\title{
EDITORIAL
}

\section{Judge the science, not the funding source}

International Journal of Obesity (2014) 38, 625; doi:10.1038/ ijo.2014.32; published online 18 March 2014

Medical research has long been engaged in debate over the influences of corporate sponsorship on research findings. These discussions are a necessary element of our scientific process. However, recently the issue of 'funding source' has taken on a life of its own, particularly in the realm of obesity research. Discussions about the merit and objectivity of the underlying science frequently take a back seat to ad hominem attacks on researchers or accusations of malicious corporate intent in the absence of any objective scientific appraisal of the research.

These discussions are based on the faulty logic that somehow direct corporate funding is inherently bias-producing in otherwise ethical researchers and that, by implication, public (for example, $\mathrm{NIH}$, USDA) and/or philanthropic (for example, RWJ, AHA) funding, by way of the intervening agency, ensures objectivity. A recent controlled study of over 500 board-certified internists found that the participant's perception of methodological quality was lower if they believed a trial was corporate sponsored even when no actual methodological difference was present. ${ }^{1}$ This suggests a bias against scientifically valid studies based solely on funding source, which could in fact negatively impact public health through out-of-hand dismissal of relevant, high-quality research.

Conversely, the implied bias favoring noncorporate trial objectivity is equally concerning as it may lead us to overlook very real potential bias from publicly funded trials. For example, it could be argued that a scientist who is funded by USDA is at similar risk for being biased in favor of sugar, corn and other agriculture-based products vs artificial sweeteners. Moreover, an often overlooked threat to objectivity is the pressure to find statistically significant results. This has been found in both corporate- and noncorporate-sponsored research. A recent examination of over 4600 scientific papers from all disciplines published internationally between 1990 and 2007 found that the frequency of positive support for hypotheses has increased $22 \%$. The author notes that this bias toward publishing positive results may influence the objectivity of the research literature both directly and also more subtly by discouraging more innovative (higher-risk) projects. ${ }^{2}$

Assuming that the well-intentioned yet misguided goal of those leveling these ad hominem attacks is to keep scientists in check and to help us avoid moral and scientific pitfalls, there is a far better model available. When the model works, the public is protected, scientific discovery and rigor supported, and advances in our knowledge achieved. At the core of the model is a safe environment that allows for transparency. This includes full disclosure of all potential conflicts of interest without fear of judgment or reprisal and peer-reviewed publication of findings with appropriate methodological detail to allow for objective analysis and scientific scrutiny. Beyond these commonly and universally held practices, more consistent application of additional tools may be useful. For example, a requirement for all research to be preregistered in Clinicaltrials.gov or a similar database. Reviewers and journal editors can be encouraged to ensure that final manuscripts are consistent with the stated $a$ priori objectives before final acceptance of manuscripts, which could further add to our protection of scientific integrity. Finally, we should all aspire to solve the intellectual property and other barriers that limit our ability to review and replicate studies on the basis of lack of access to primary data sources in some corporate trials. Although these barriers are complex and beyond the scope of this paper, we need to begin to find solutions that will enhance the ways scientific process can be used to be the judge of all science.

In summary, what are the best practices for ensuring a strong, unbiased body of obesity research? Certainly not refusing funding from those who wish to collaborate with scientists in becoming part of the solution; absolutely not by launching unsubstantiated attacks on reputable scientists with longstanding records of ethical conduct and meaningful scientific contribution; rather, it is first by giving funding source its proper position among many possible and equally important threats to objectivity and implementing safeguards to protect against such bias (and worse yet malfeasance). Second, we need to redouble our efforts to adhere to the basic principles of good science like reproducibility, replicability and other core evaluative procedures that ensure objective and reliable scientific reporting. Finally, we need to work toward open access to data regardless of its source. This will require the cooperation of those in the scientific community and among potential sources of funding. Ultimately, this type of transparency regardless of funding source will deliver a more robust and complete body of evidence. In short, scientists need to practice good science, sponsors must commit to transparency and noninfluence, media needs to practice responsible scientific journalism, and we all need to base our evaluations on scientific data and not on predetermined opinions rooted in our own emotion-laden bias for or against specific funding sources.

\section{CONFLICT OF INTEREST}

Dr Martin Binks reports the following potential conflicts of interest: Dr Binks is sole proprietor of Binks Behavioral Health PLLC; he has also received financial compensation from: The Obesity Society (Communications \& Social Media Consultant), 2011-present; Guidepoint Global Consulting, 2007-present; AbbVie Men's Health Initiative, 2012; Everyday Health Inc., 2006-2011; Evolution Health Systems, 2006-2011. Dr Binks also currently serves in the following volunteer roles: The Obesity Society, Secretary Treasurer \& Development Chair. No funding from any source is directly associated with the development of this manuscript.

\section{Binks ${ }^{1,2}$ \\ ${ }^{1}$ Department of Nutritional Sciences, Texas Tech University, Lubbock, TX, USA and ${ }^{2}$ Binks Behavioral Health, PLLC, Hillsborough, NC, USA E-mail: m.binks@ttu.edu}

\section{REFERENCES}

1 Kesselheim AS, Robertson CT, Myers JA, Rose SL, Gillet V, Ross KM et al. A randomized study of how physicians interpret research funding disclosures. N Engl J Med 2012; 367: 1119-1127.

2 Fanelli D. Negative results are disappearing from most disciplines and countries. Scientometrics 2012; 90 : 891-904. 